\title{
Technology Transfer at the University of Basel
}

\author{
Bruno H. Dalle Carbonare*
}

\begin{abstract}
The University of Basel is Switzerland's oldest university. Since 1996 the University has enjoyed an 'autonomous status' and is governed by a 'University Council'. Thus, the five hundred years of old academic tradition demonstrated a pronounced willingness to change. With its seven faculties, the University of Basel covers all academic fields; however, two areas will be focussed on: life sciences and culture. The Office of Technology Transfer (OTT) of the University of Basel was created in 1997. In addition to the University of Basel, in Northwestern Switzerland the Universities of Applied Sciences Basel (FHBB), Aargau (FHA), and Solothurn (FHSO) as well as the University Hospitals of Basel (KBS) have also entirely or partially entrusted their technology transfer to the OTT of the University of Basel. Over the past years, the OTT extended its services beyond the conventional activities of technology transfer involving transfer of intellectual property, transfer negotiations and follow-up, as well as active scouting for exploitable know-how and research results. The successful record of the OTT is attributable to a number of success factors: greater participation by researchers in technology transfer, higher productivity as a result of the innovative public-private partnership (PPP) between a private company specialized in technology transfer and the University of Basel and favourable financial incentives for the inventors. The rapidly growing number of transactions shows the strategic significance of technology transfer and its various aspects for all stakeholders.
\end{abstract}

Keywords: Innovation transfer - Technology scouting · Technology transfer - University Hospital of Basel · University of Applied Sciences · University of Basel

\section{Introduction}

The term 'technology transfer' describes the transfer and marketing of research results produced at universities to society at large, and especially to companies. Technology transfer is therefore of relevance to all universities, university-type establishments and university members. For this reason, technology transfer is increasingly being regarded, along with teaching and research, as the third main purpose of universities.
Technology transfer out of public universities is a public duty. The aim of technology transfer is therefore not primarily to generate additional income for the universities, including achieving a high level of self-financing of the Office of Technology Transfer (OTT). There are few universities in the world where technology transfer and the running of an OTT for the university are financially self-supporting. The aim of technology transfer is rather to provide services for society at large. Research results generated at universities are achieved with tax money, while society in turn receives new products and services whose production and performance create jobs and added value. Technology transfer therefore produces wide-ranging benefits and commands an increasingly important strategic position.

\section{The Swiss National Technology Transfer Environment}

Horrespondence: Dr. B.H. Dalle Carbonare

Office of Technology Transfer

University of Basel

Petersgraben 35

$\mathrm{CH}-4003$ Basel

Tel.: + 41612672731

Fax: +41612670997

E-Mail: Bruno.DalleCarbonare@unibas.ch technology ably in Switzerland. Politicians in particular hoped and expected that technology transfer would contribute to greater inno- vation, economic growth and added value resulting in more jobs for highly qualified members of universities [1]. At the same time, there were attempts to improve the general conditions for technology transfer in Switzerland:

- During the discussion of the position paper on 'Education, research and technology for the 2004-2007 legislative period', the national parliament also looked closely at technology transfer and approved a basic budget of CHF 16 million for innovation and the exploitation of know-how. Technology transfer at universities can expect to receive a substantial proportion of this budget.

- The Swiss University Conference (SUK/CUS) has arranged for a broad study developing the national strategy regarding technology transfer in Switzerland and is considering issuing guidelines for technology transfer to universities in Switzerland [2].

- Finally, towards the end of 2003, the Swiss Technology Transfer Association (swiTT) was formed, made up of members of OTTs from different universities.

Representatives of the University of Basel and its OTT participated in all these 
processes and were at all times heavily involved. It can therefore be taken for granted that these developments will have a positive effect on technology transfer at the University of Basel.

\section{The Office of Technology Trans- fer (OTT) at the University of Basel}

The University of Basel is Switzerland's oldest university. Since 1996 the University has enjoyed an 'autonomous status' and is governed by a 'University Council'. Thus, the five hundred years of old academic tradition demonstrated a pronounced willingness to change. With its seven faculties, the University of Basel covers all academic fields; however, two areas will be focussed on: life sciences and culture. Life sciences are anchored in the world-famous Biocenter and are today complemented by acknowledged competencies in nanosciences and strong clinical-biological research.

The OTT of the University of Basel acts as a central point of contact and service, offering advice and support to university members in the transfer of their know-how and research results. Accordingly, the marketing of work carried out by the university is currently the OTT's main activity (push). In future, the OTT will also initiate activities for the stimulation of demand for technology transfer (pull) among companies.

2003 was the most successful year for the OTT of the University of Basel since it was created in 1997. All the essential indicators for success of the OTT improved considerably. Taking into account the technology transfer potential of the associated universities, it can now be assumed that the OTT of the University of Basel is one of Switzerland's best performing OTTs, placing it even among the $10 \%$ most successful OTTs in the world [3].

\subsection{Expansion of the Operational Range}

The OTT of the University of Basel is becoming a regional centre of competence for technology transfer in Northwestern Switzerland. In 2003, the following universities and public research institutes entrusted their technology transfer to the OTT of the University of Basel:

- University of Basel

- University Hospital of Basel (KBS)

- University of Applied Sciences Basel (FHBB)

- University of Applied Sciences Aargau (FHA)

Since 1 January 2004 the University of Applied Sciences Solothurn (FHSO) also uses the services of the OTT. Despite the increase in the number of universities looked after by the OTT, the University of Basel remains its highest priority.

\subsection{Expansion of the Scope of Activities}

The transfer of research results is a complex process that rarely takes place in a straightforward manner or according to a precise timescale known in advance, but in practice involves a number of formalities. Nevertheless, it is true to say that the added value chain in technology transfer comprises the following six tasks:

1. Transfer culture, or determination of the transfer policy, guidelines and framework conditions at the university;

2. Transfer initiation, or identifying transfer possibilities (scouting), searching for partners for cooperation in a transfer project;

3. Transfer of intellectual property, or evaluation and, if applicable, protection of inventions/patents;

4. Transfer negotiations, or building up a negotiation position for the transfer, negotiating cooperation and licence agreements, resolving conflicts of interest;

5. Transfer follow-up, or monitoring of transfer partners as well as support of start-ups from the universities;

6. Transfer cooperation, or support in cooperation between the University and Universities of Applied Sciences in development projects and regional and national cooperation in technology transfer.

Up until the end of 2001, the OTT concentrated primarily on the 'conventional' activities of technology transfer, that is to say the transfer of intellectual property (task 3), carrying out transfer negotiations (task 4) and a certain amount of transfer follow-up (task 5).

Since the beginning of 2002, the scope of work carried out by the OTT has been substantially extended, the OTT carrying out additional work not done before. This includes work in the forefront of technology transfer, specifically transfer initiation work (task 2). Here, the University of Basel conducted active scouting and identification of potential transfer projects by routine contacts with professors and other university members. The 'Technology Scouting' pilot project confirmed that the University of Basel had considerable potential in terms of know-how and research results that could be used for technology transfer. On the basis of a sample of 20 institutions and research groups of the University of Basel, it was possible to identify various research results suitable for transfer. The discussions revealed a direct need for OTT services, such as advice/support in contractual agreements.
With a certain time lag, scouting therefore has exerted a direct and positive effect on the number of projects from the University of Basel managed by the OTT.

In 2003 the OTT extended its activities to transfer follow-up (task 5) and transfer cooperation (task 6) work. Particular mention is made here of the 'Innovation Transfer' project [4]. Supported by the Vice-Chancellor for Research, results and technologies originating from basic research are developed together with the Universities of Applied Sciences of Northwestern Switzerland (FHBB, FHSO, FHA) in a target-specific manner. The aim is to develop products all the way to near market introduction. In 2003, a total of five projects was carried out under the framework of 'Innovation Transfer'; one of these projects being financed by a US investor.

'Innovation Transfer' between the University of Basel and the Universities of Applied Sciences of Northwestern Switzerland is exemplary and almost unique in this country. This has also led to support for individual projects in the 'Innovation Transfer' field by the Commission for Innovation and Technology (CTI) of the Federal Office for Professional Education and Technology (OPET).

Since the spring of 2003, the OTT has, thanks to the use of a new database, started to market inventions systematically around the world, potentially interested companies being contacted directly by email. This proactive approach has considerably increased the commercial use of research results from the University of Basel.

\subsection{Growing Number of Projects}

After a significant increase in the number of projects managed by the OTT in 2002, this trend continued in 2003 and became even more pronounced. Overall, 193 new projects were taken on by the OTT in 2003, nearly twice as many as in 2002. Including the 124 projects still pending from the previous years 1998-2002, a total of 317 projects were therefore being actively managed by the OTT in 2003.

Only a small fraction of these projects were connected with the founding of new companies by university members and start-ups. The reason for this was that only two start-ups from the University of Basel were founded in 2003. This is not because of a low motivation by university members or lack of interest in forming start-ups by universities or the OTT, far from it. The first reason for the comparatively small number of start-ups is the scarcity of facilities and measures for the active promotion and support of start-ups in Northwestern Switzerland. As a result, 
founders find it especially difficult to create new companies in the Basel region. Secondly, the potential for founding startups is limited. As a result, the vast majority of transfers managed by the OTT are with existing companies in Switzerland and abroad.

\subsection{Contracts with Third Parties}

Of the total of 317 projects managed, 256 involved working on, arranging and negotiating contractual agreements such as services/orders (16), R\&D collaborations (50), EU contracts (12), inter-institutional agreements between universities (20), licence agreements (55), intellectual property right transfer contracts or IP transfer (15), confidentiality agreements or CDAs (43) and material transfer agreements or MTAs (31), consultancy agreements (1), gifts/sponsorship (3) and other agreements with third parties (10). In comparison, in the previous two years, only 25 (2001) and 80 (2002) projects respectively included work on contractual agreements (Fig.).

In order to financially assess the contracts managed by the OTT and signed in 2003, the corresponding contract value was determined according to monetary or non-monetary criteria. The monetary payment flows and the value of other items such as equipment and materials covered under these contracts were added up. Thus, the total value is estimated at CHF 126 million, CHF 78 million of which came from EU consortium agreements.

\subsection{Patents}

A total of 45 of the 193 new projects taken on in 2003 involved activities in the field of patent property rights such as invention disclosures (31), and patent and commercial evaluations resulting in patent applications (11). Compared to the previous year, this is a doubling of the patenting activities of the OTT.

\subsection{Marketing}

A total of 27 of the 317 projects involved the active marketing of research results and technologies of the University of Basel. Using internet searches, more than 1000 potential exploitation partners around the world were identified for these projects, and these were subsequently contacted with project-specific 'Project Summaries'.

This procedure generated around 5000 communications with potential partners. This marketing strategy led, in 2003, to the signing of five licence and one option agreement, resulting so far in total royalties of around CHF 250'000. There is the possibility of millions more in royalties if industrial partners take up the pending options.

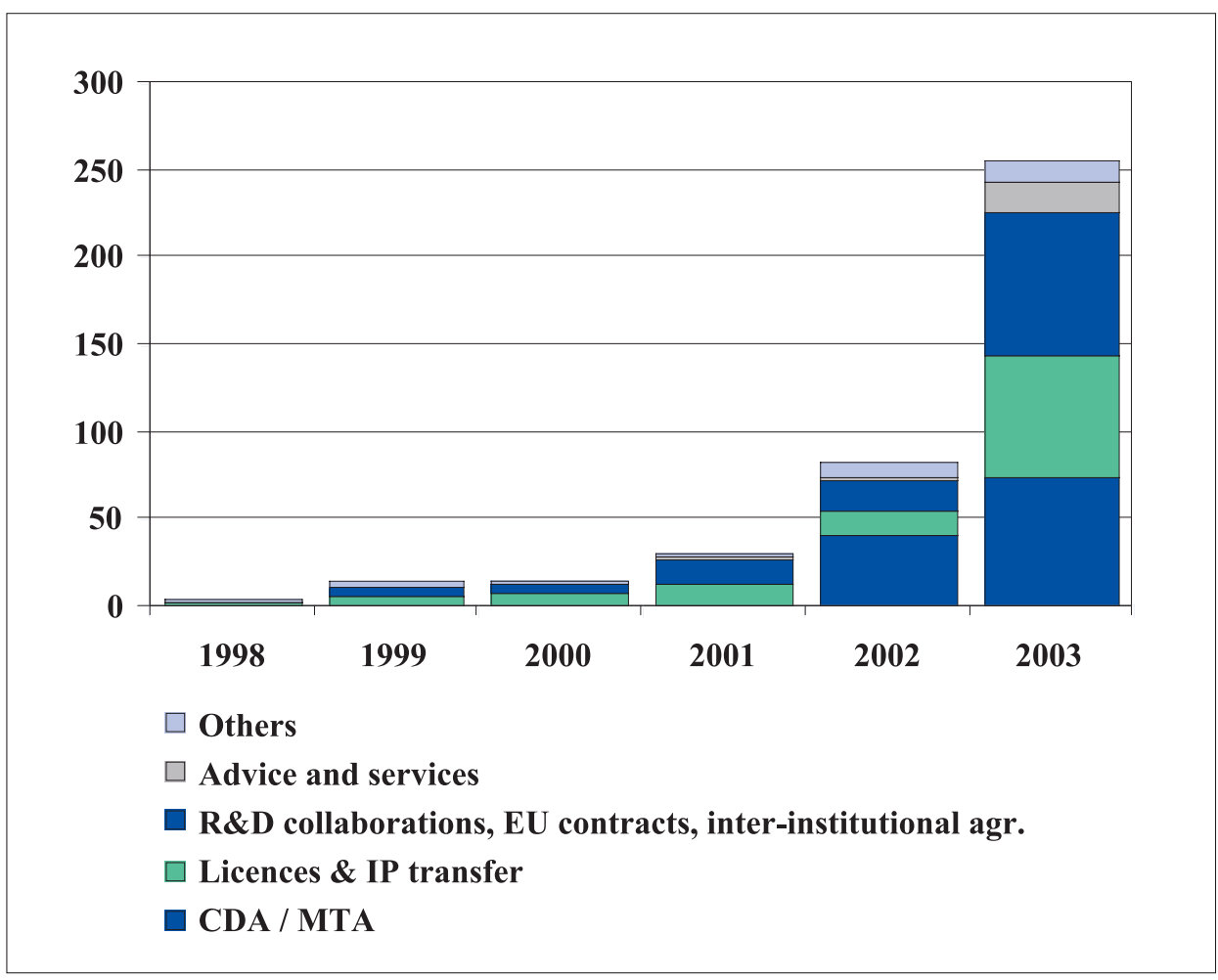

Fig. Projects managed by the OTT

\section{Success Factors of Technology Transfer at the University of Basel}

The progress made by the OTT can be attributed to the following factors:

\subsection{Expert Staff}

Since technology transfer is primarily a 'people business', the persons working in the OTT require skills and experience in the following fields: the sciences, particularly life sciences, patents and licensing, law, marketing, business management, as well as administration. Some of them have special expertise that is very hard to find in the market but is crucial for the quality of the work carried out by the OTT.

In view of the increased workload, the staff of the OTT was increased from 2.25 full time employees to 2.75 in 2003 of which about $20 \%$ was financed by the other universities associated with the OTT.

\subsection{Greater Participation by Researchers in Technology Transfer}

Technology transfer would be impossible without researchers having the necessary interest in technology transfer and therefore wishing to be involved in it. The increasing number of technology transfer projects in 2003 is mainly due to the fact that, at the University of Basel, but also at the University Hospital of Basel (KBS), FHBB and FHA, more and more re- searchers have looked at technology transfer and decided to hand their projects over to the OTT. These researchers are not just from science or engineering, which are traditionally more involved in technology transfer, but increasingly also from other disciplines. This is another indication of the increasing acceptance of technology transfer and its recognition among the academic community.

\subsection{Financial Arrangements}

The work carried out by the OTT is basically free for members of the University of Basel and the other universities associated with the OTT. As a result of the larger number of technology transfer projects, however, the costs for patents and in particular for repayments of financial income from successful transfers to the institutions of the universities and inventors is increasing. According to the rules established by the universities, $40 \%$ thereof goes to the inventor(s) and 30\% each to the research group/institute and the parent university. The level of self-financing of the OTT is $70 \%$. The operating deficit of the OTT of the University of Basel has remained relatively constant over the last few years, while the 'turnover' has increased considerably. Therefore, despite the fact that the OTT carries out more and more work, it is not more expensive for the university than before. Thus, the increase in technology transfer work was at no extra cost. This is of benefit to all those involved. 


\subsection{High Productivity}

The number of technology transfer projects managed per full-time post was dramatically increased from 24 in 2001 to 70 in 2003. This increase by $300 \%$ reflects the great efforts to improve the efficiency of the operational procedures in spite of the fact that not only the number of projects grew, but their complexity also increased significantly (numerous universities involved, inventions under greater competition, negotiations with multinational companies, etc.).

\subsection{Innovative Organizational Model}

One of the main reasons for the current performance of the OTT is its innovative organizational model. Due to a successful public-private partnership (PPP) in force since the middle of 2001, operational management and the provision of services of the OTT of the University of Basel has been handed over to the company BDC $\mathrm{GmbH}$ on the basis of a service agreement, while the strategic management of the OTT is retained by the Vice-Chancellor for Research of the University of Basel. As a result, a commercial attitude and approach has been introduced at the OTT which underwent a 'business reengineering process' to be able to provide efficient services. In addition to the introduction of modern management instruments and information technology, BDC can provide the specialists necessary for each job at the OTT in a targeted manner and on a part-time basis thereby enabling access to the knowledge and experience of about ten specialists.

As a result of the efforts to expand geographical area and scope of work, the status of technology transfer has improved considerably in Northwestern Switzerland. With constant financial expenditure by universities associated with the OTT for technology transfer, the output has increased. As a consequence of technology transfer, new companies and jobs for university members have been created in the region.

\section{Outlook}

There is still much room for expanding the activities of the OTT. In particular, as shown by the results achieved at the University of Basel in the 'Technology Scouting' pilot project, there is still great potential in this domain.

Thus, the OTT will continue to build on the factors for success described above and strive to satisfy the growing demand among university members and the local economy for its services.

Received: October 20, 2004

[1] Swiss Federal Office of Economics (EVD), 'InnoNation Schweiz' ('Swiss
InnoNation'), June 2003 and EVD, 'Determinanten des Schweizer Wirtschaftswachstums und Ansatzpunkte für eine wachstumsorientierte Wirtschaftspolitik' ('Factors determining Switzerland's economic growth and starting points for a growth-orientated economic policy'), Bern, March 2003.

[2] W. Zinkl, H. Huber, COGIT Basel, 'Strategie für den WTT an den Hochschulen in der Schweiz' ('Strategy for technology transfer at universities in Switzerland'), Basel, April 2003, study carried out on behalf of SUK.

[3] Annual Report for the Association of university Technology Managers (AUTM), USA (Survey 2000), and OECD study 'Turning Science into Business, Patenting and Licensing at Public Research Organisations' (OECD 2003).

[4] D. Vonder Mühll, H. Flaadt, M. Hoh, L. Jäger, A. Sandor, S. Schwarz, F. Stapf, D. Gygax, 'Innovation transfer-pilot project between cantonal universities and universities of applied science in the field of research and development on the example of the University of Basel and University of Applied Sciences Basel', April 2003; study carried out with financial support of the University of Basel and universities of applied sciences Basel. 